Research Article

\title{
The Recursive Properties of the Error Term of the Fourth Power Mean of the Generalized Cubic Gauss Sums
}

\author{
Zhang Jin ${ }^{1}$ and Zhang Jiafan $\mathbb{D}^{2}$ \\ ${ }^{1}$ School of Information Engineering, Xi'an University, Xi'an 710065, China \\ ${ }^{2}$ School of Mathematics, Northwest University, Xi'an 710127, China \\ Correspondence should be addressed to Zhang Jiafan; zhangjiafan@stumail.nwu.edu.cn
}

Received 11 March 2021; Revised 23 March 2021; Accepted 26 March 2021; Published 10 April 2021

Academic Editor: Tingting Wang

Copyright ( $) 2021$ Zhang Jin and Zhang Jiafan. This is an open access article distributed under the Creative Commons Attribution License, which permits unrestricted use, distribution, and reproduction in any medium, provided the original work is properly cited.

In this paper, we use the analytic methods and the properties of the classical Gauss sums to study the properties of the error term of the fourth power mean of the generalized cubic Gauss sums and give two recurrence formulae for it.

\section{Introduction}

For any integer $q \geq 2$ and any Dirichlet character $\chi \bmod q$, the definition of the classical Gauss sums $G(m, \chi ; q)$ is

$$
G(m, \chi ; q)=\sum_{a=1}^{q} \chi(a) e\left(\frac{m a}{q}\right)
$$

where $m$ is an integer and $e(y)=e^{2 \pi i y}$.

This sum and its properties are of great significance to the analytic number theory, and many number theory problems are closely related to them. Therefore, it is necessary to study the various properties of $G(m, \chi ; q)$ and related sums. In this paper, we consider the generalized $k$-th Gauss sums $G(m, k, \chi ; q)$, which is defined as follows:

$$
G(m, k, \chi ; q)=\sum_{a=1}^{q} \chi(a) e\left(\frac{m a^{k}}{q}\right),
$$

where $k$ is any positive integer and $m$ is an integer with $(m, q)=1$.

It is clear that this sum is a generalization of the classical Gauss sums $G(m, \chi ; q)$. In fact, $G(m, 1, \chi ; q)=G(m, \chi ; q)$. Of course, the value of $G(m, k, \chi ; q)$ is irregular as $\chi$ varies. However, some scholars have found that $G(m, k, \chi ; q)$ has good value distribution properties in some problems of weighted mean value, even if we can get their exact calculation formulae for some $2 k$ th power mean. In addition, there are some good upper bound estimates for $|G(m, k, \chi ; q)|$. For example, for any integer $n$ with $(n, q)=1$, from the general result of Cochrane and Zheng [1], we can deduce

$$
|G(m, k, \chi ; q)| \leq 2^{\omega(q)} q^{(1 / 2)},
$$

where $\omega(q)$ denotes the number of distinct prime divisors of $q$. The case that $q$ is a prime is due to Weil [2].

For $k=2$, by the results of Zhang [3], let $n$ be any integer with $(n, p)=1$, and there are the following two identities:

$$
\begin{array}{ll}
\frac{1}{p-1} \sum_{\chi \bmod p}|G(n, 2, \chi ; p)|^{4}= \begin{cases}3 p^{2}-6 p-1+4\left(\frac{n}{p}\right) \sqrt{p}, & \text { if } p \equiv 1 \bmod 4, \\
3 p^{2}-6 p-1, & \text { if } p \equiv 3 \bmod 4,\end{cases} \\
\frac{1}{p-1} \sum_{\chi \bmod p}|G(n, 2, \chi ; p)|^{6}=10 p^{3}-25 p^{2}-4 p-1, & \text { if } p \equiv 3 \bmod 4,
\end{array}
$$


where $(* / p)$ denotes the Legendre symbol modulo $p$.

Zhang and Liu [4] have studied the sum

$$
\sum_{\chi \bmod p}|G(n, 3, \chi ; q)|^{4}
$$

and obtained the following calculation formula: where $p$ is a prime with $3 \mid p-1$ and $U=\sum_{a=1}^{p} e\left(a^{3} / p\right)$ is a real constant.

However, the value of $U$ was not given in [4], and the form of $U$ was not concise. Now, for any integer $1 \leq m \leq p-1$, we let

$$
E(m, p)=\frac{1}{p-1} \sum_{\chi \bmod p}|G(m, 3, \chi ; p)|^{4}-5 p^{2}+13 p+1 .
$$

In this paper, we use the analytic methods and the properties of the classical Gauss sums to study the calculating problem of the $n$th power mean of $E(m, p)$ and give two recurrence formulae for it. That is, we shall prove the following main results.

Theorem 1. Let $p$ be an odd prime with $3 \mid p-1$. Then, for any positive integer $n$ and integer $m$ with $(m, p)=1$, we have the third-order linear recurrence formula:

$$
E^{n}(m, p)=U(p, d) \cdot E^{n-3}(m, p)+V(p, d) \cdot E^{n-2}(m, p),
$$

where $U(p, d)$ and $V(p, d)$ are defined as

$$
\begin{aligned}
U(p, d)= & 64 d p+\left(d^{5}-12 d^{4}+60 d^{3}-160 d^{2}+240 d-384\right) p^{2} \\
& -\left(5 d^{3}-48 d^{2}+180 d-320\right) p^{3}+(5 d-24) p^{4} \\
V(p, d)= & 3 p \cdot\left(p^{2}+8 p-4 d p+4 d^{2}-16 d+16\right)
\end{aligned}
$$

and $4 p=d^{2}+27 b^{2}$, where $d$ is uniquely determined by $d \equiv 1 \bmod 3($ see $[5])$.

Theorem 2. Let $p$ be an odd prime with $3 \mid p-1$. Then, for any positive integer $n \geq 3$, we have the recurrence formula

$$
\begin{aligned}
\sum_{m=1}^{p-1} E^{n}(m, p)= & U(p, d) \sum_{m=1}^{p-1} E^{n-3}(m, p) \\
& +V(p, d) \sum_{m=1}^{p-1} E^{n-2}(m, p),
\end{aligned}
$$

where the first three terms of $\sum_{m=1}^{p-1} E^{n}(m, p)$ are

$$
\begin{aligned}
& \sum_{m=1}^{p-1} E(m, p)=0, \\
& \sum_{m=1}^{p-1} E^{2}(m, p)=2 p(p-1)(p-2 d+4)^{2}, \\
& \sum_{m=1}^{p-1} E^{3}(m, p)=(p-1) U(p, d) .
\end{aligned}
$$

Theorem 3. Let $p$ be an odd prime with $3 \mid p-1$. Then, for any positive integer $n \geq 1$, we have the third-order linear recurrence formula

$$
\sum_{m=1}^{p-1} E^{-n}(m, p)=\frac{1}{U(p, d)} \cdot \sum_{m=1}^{p-1} E^{-(n-3)}(m, p)-\frac{V(p, d)}{U(p, d)} \cdot \sum_{m=1}^{p-1} E^{-(n-1)}(m, p)
$$


where the initial values of $\sum_{m=1}^{p-1} E^{-n}(m, p)$ are

$$
\begin{aligned}
& \sum_{m=1}^{p-1} E^{-1}(m, p)=\frac{2 p(p-1)(p-2 d+4)^{2}-(p-1) V(p, d)}{U(p, d)}, \\
& \sum_{m=1}^{p-1} E^{-2}(m, p)=\frac{(p-1) V^{2}(p, d)-2 p(p-1)(p-2 d+4)^{2} V(p, d)}{U^{2}(p, d)}, \\
& \sum_{m=1}^{p-1} E^{-3}(m, p)=\frac{p-1}{U(p, d)}+\frac{2 p(p-1)(p-2 d+4)^{2} V^{2}(p, d)-(p-1) V^{3}(p, d)}{U^{3}(p, d)} .
\end{aligned}
$$

Taking $n=4$ in Theorem 3, we may immediately deduce the following corollary.
Corollary 1. Let $p$ be an odd prime with $3 \mid p-1$; then, we have the identity

$$
\begin{aligned}
& \frac{1}{p-1} \sum_{m=1}^{p-1} \frac{1}{|E(m, p)|^{4}} \\
& =\frac{2 p(p-2 d+4)^{2}-2 V(p, d)}{U^{2}(p, d)}+\frac{V^{4}(p, d)-2 p(p-2 d+4)^{2} V^{3}(p, d)}{U^{4}(p, d)} . \\
& \tau^{3}(\lambda)+\tau^{3}(\bar{\lambda})=d p,
\end{aligned}
$$

\section{Several Lemmas}

In this section, we give three lemmas which are necessary in the proofs of our theorems. In the process of proving our lemmas, we need some knowledge of the analytic number theory; all of which can be found in [6-8], so it is not necessary to repeat them here.

Lemma 1. Let $p$ be an odd prime with $p \equiv 1 \bmod 3$. Then, for any third-order character $\lambda \bmod p$, we have where $d$ is uniquely determined by $4 p=d^{2}+27 b^{2}$ and $d \equiv 1 \bmod 3$.

Proof. This result can be found in [9] or [10].

Lemma 2. Let $p$ be an odd prime with $3 \mid p-1$. Then, for any cubic character $\lambda \bmod p$, we have the identities

$$
\begin{aligned}
E(m, p) & =\bar{\lambda}(m)\left(\sum_{a=1}^{p-1} \bar{\lambda}\left(a^{3}-1\right)\right)^{2} \cdot \tau(\lambda)+\lambda(m)\left(\sum_{b=1}^{p-1} \lambda\left(b^{3}-1\right)\right)^{2} \cdot \tau(\bar{\lambda}) \\
& =\bar{\lambda}(m) \tau(\lambda)\left(\frac{\tau^{3}(\bar{\lambda})}{p}-2\right)^{2}+\lambda(m) \tau(\bar{\lambda})\left(\frac{\tau^{3}(\lambda)}{p}-2\right)^{2} .
\end{aligned}
$$

Proof. For any integer $1 \leq a \leq p-1$, it is easy to show that

$$
1+\lambda(a)+\lambda^{2}(a)= \begin{cases}3, & \text { if } a \text { is a cubic residue } \bmod p \\ 0, & \text { otherwise }\end{cases}
$$

From the properties of the cubic character modulo $p$, we have

$$
\begin{aligned}
\lambda^{2} & =\bar{\lambda}, \\
\lambda(-1) & =1, \\
\overline{\tau(\lambda)} & =\tau(\bar{\lambda}) .
\end{aligned}
$$


So, we have the identity

$$
\begin{aligned}
\sum_{a=1}^{p-1} \lambda\left(a^{3}-1\right) & =\frac{1}{\tau(\bar{\lambda})} \sum_{b=1}^{p-1} \sum_{a=1}^{p-1} \bar{\lambda}(b) e\left(\frac{b\left(a^{3}-1\right)}{p}\right) \\
& =\frac{1}{\tau(\bar{\lambda})} \sum_{b=1}^{p-1} \bar{\lambda}(b) e\left(\frac{-b}{p}\right) \sum_{a=1}^{p-1}(1+\lambda(a)+\bar{\lambda}(a)) e\left(\frac{b a}{p}\right) \\
& =\frac{1}{\tau(\bar{\lambda})} \sum_{b=1}^{p-1} \bar{\lambda}(b) e\left(\frac{-b}{p}\right)(-1+\bar{\lambda}(b) \tau(\lambda)+\lambda(b) \tau(\bar{\lambda}) \\
& =\frac{1}{\tau(\bar{\lambda})}\left(-\lambda(-1) \tau(\bar{\lambda})+\tau(\lambda) \sum_{b=1}^{p-1} \lambda(b) e\left(\frac{-b}{p}\right)+\tau(\bar{\lambda}) \sum_{b=1}^{p-1} e\left(\frac{-b}{p}\right)\right) \\
& =\frac{1}{\tau(\bar{\lambda})}\left(-\tau(\bar{\lambda})+\tau^{2}(\lambda)-\tau(\bar{\lambda})\right)=\frac{\tau^{3}(\lambda)}{p}-2 .
\end{aligned}
$$

Therefore,

$$
\begin{aligned}
& E(m, p)= \bar{\lambda}(m) \tau(\lambda)\left(\sum_{a=1}^{p-1} \bar{\lambda}\left(a^{3}-1\right)\right)^{2}+\lambda(m) \tau(\bar{\lambda})\left(\sum_{a=1}^{p-1} \lambda\left(a^{3}-1\right)\right)^{2} \\
&= \bar{\lambda}(m) \tau(\lambda)\left(\frac{\tau^{3}(\bar{\lambda})}{p}-2\right)^{2}+\lambda(m) \tau(\bar{\lambda})\left(\frac{\tau^{3}(\lambda)}{p}-2\right)^{2} \cdot \\
& E^{3}(m, p)=U(p, d)+V(p, d) \cdot E(m, p),
\end{aligned}
$$

This proves Lemma 2.

Lemma 3. Let $p$ be an odd prime with $3 \mid p-1$. Then, for any cubic character $\lambda$ modulo $p$, we have the identity where

$$
\begin{aligned}
U(p, d)= & 64 d p+\left(d^{5}-12 d^{4}+60 d^{3}-160 d^{2}+240 d-384\right) p^{2} \\
& -\left(5 d^{3}-48 d^{2}+180 d-320\right) p^{3}+(5 d-24) p^{4} \\
V(p, d)= & 3 p \cdot\left(p^{2}+8 p-4 d p+4 d^{2}-16 d+16\right)
\end{aligned}
$$

and $E(m, p)$ is defined as the same as in Lemma 2. 
Proof. From Lemma 2, we have

$$
\begin{aligned}
E^{3}(m, p)= & \left(\bar{\lambda}(m) \tau(\lambda)\left(\frac{\tau^{3}(\bar{\lambda})}{p}-2\right)^{2}+\lambda(m) \tau(\bar{\lambda})\left(\frac{\tau^{3}(\lambda)}{p}-2\right)^{2}\right)^{3} \\
= & \left(\tau(\lambda)\left(\frac{\tau^{3}(\bar{\lambda})}{p}-2\right)^{2}\right)^{3}+\left(\tau(\bar{\lambda})\left(\frac{\tau^{3}(\lambda)}{p}-2\right)^{2}\right)^{3} \\
& +3 p \cdot\left(\frac{\tau^{3}(\bar{\lambda})}{p}-2\right)^{2}\left(\frac{\tau^{3}(\lambda)}{p}-2\right)^{2} \cdot E(m, p) .
\end{aligned}
$$

Note that $\tau(\lambda) \tau(\bar{\lambda})=p$, and from (17), we have

$$
\begin{aligned}
\tau^{6}(\lambda)+\tau^{6}(\bar{\lambda}) & =\left(\tau^{3}(\lambda)+\tau^{3}(\bar{\lambda})\right)^{2}-2 \tau^{3}(\lambda) \tau^{3}(\bar{\lambda}) \\
& =d^{2} p^{2}-2 p^{3} .
\end{aligned}
$$

Therefore,

$$
\begin{aligned}
\left(\frac{\tau^{3}(\bar{\lambda})}{p}-2\right)^{2}\left(\frac{\tau^{3}(\lambda)}{p}-2\right)^{2} & \\
= & \left(4-\frac{4 \tau^{3}(\bar{\lambda})}{p}+\frac{\tau^{6}(\bar{\lambda})}{p^{2}}\right) \cdot\left(4-\frac{4 \tau^{3}(\lambda)}{p}+\frac{\tau^{6}(\lambda)}{p^{2}}\right) \\
= & 16-\frac{16}{p}\left(\tau^{3}(\lambda)+\tau^{3}(\bar{\lambda})\right)+\frac{4}{p^{2}}\left(\tau^{6}(\lambda)+\tau^{6}(\bar{\lambda})\right)+\frac{16}{p^{2}} \tau^{3}(\lambda) \tau^{3}(\bar{\lambda}) \\
& -\frac{4}{p^{3}}\left(\tau^{3}(\lambda) \tau^{6}(\bar{\lambda})+\tau^{3}(\bar{\lambda}) \tau^{6}(\lambda)\right)+\frac{1}{p^{4}} \tau^{6}(\lambda) \tau^{6}(\bar{\lambda}) \\
= & 16-\frac{16}{p} \cdot d p+\frac{4}{p^{2}} \cdot\left(d^{2} p^{2}-2 p^{3}\right)+\frac{16}{p^{2}} \cdot p^{3}-\frac{4}{p^{3}} \cdot p^{3} \cdot d p+\frac{1}{p^{4}} \cdot p^{6} \\
= & p^{2}+8 p-4 d p+4 d^{2}-16 d+16 .
\end{aligned}
$$


In addition,

$$
\begin{aligned}
\left(\tau(\lambda)\left(\frac{\tau^{3}(\bar{\lambda})}{p}-2\right)^{2}\right)^{3}= & \tau^{3}(\lambda)\left(\frac{\tau^{3}(\bar{\lambda})}{p}-2\right)^{6} \\
= & \tau^{3}(\lambda)\left(\frac{\tau^{18}(\bar{\lambda})}{p^{6}}+36 \frac{\tau^{12}(\bar{\lambda})}{p^{4}}+144 \frac{\tau^{6}(\bar{\lambda})}{p^{2}}+64-12 \frac{\tau^{15}(\bar{\lambda})}{p^{5}}\right. \\
& \left.+24 \frac{\tau^{12}(\bar{\lambda})}{p^{4}}-16 \frac{\tau^{9}(\bar{\lambda})}{p^{3}}-144 \frac{\tau^{9}(\bar{\lambda})}{p^{3}}+96 \frac{\tau^{6}(\bar{\lambda})}{p^{2}}-192 \frac{\tau^{3}(\bar{\lambda})}{p}\right) \\
= & 64 \tau^{3}(\lambda)+240 p \tau^{3}(\bar{\lambda})-160 \tau^{6}(\bar{\lambda})+\frac{60}{p} \tau^{9}(\bar{\lambda}) \\
& -\frac{12}{p^{2}} \tau^{12}(\bar{\lambda})+\frac{1}{p^{3}} \tau^{15}(\bar{\lambda})-192 p^{2} .
\end{aligned}
$$

Using the method similar to (24), we obtain

$$
\begin{aligned}
& \tau^{9}(\lambda)+\tau^{9}(\bar{\lambda})=\left(\tau^{6}(\lambda)+\tau^{6}(\bar{\lambda})\right)\left(\tau^{3}(\lambda)+\tau^{3}(\bar{\lambda})\right)-p^{3}\left(\tau^{3}(\lambda)+\tau^{3}(\bar{\lambda})\right) \\
= & d^{3} p^{3}-3 d p^{4} \\
& \tau^{12}(\lambda)+\tau^{12}(\bar{\lambda})=d^{4} p^{4}-4 d^{2} p^{5}+2 p^{6} \\
& \tau^{15}(\lambda)+\tau^{15}(\bar{\lambda})=d^{5} p^{5}-5 d^{3} p^{6}+5 d p^{7}
\end{aligned}
$$

Combining formulae (23)-(27), we have

$$
\begin{aligned}
E^{3}(m, p)= & 64\left(\tau^{3}(\lambda)+\tau^{3}(\bar{\lambda})\right)+240 p\left(\tau^{3}(\bar{\lambda})+\tau^{3}(\lambda)\right)-160\left(\tau^{6}(\bar{\lambda})+\tau^{6}(\lambda)\right) \\
& +\frac{60}{p}\left(\tau^{9}(\bar{\lambda})+\tau^{9}(\lambda)\right)-\frac{12}{p^{2}}\left(\tau^{12}(\bar{\lambda})+\tau^{12}(\lambda)\right)+\frac{1}{p^{3}}\left(\tau^{15}(\bar{\lambda})+\tau^{15}(\lambda)\right) \\
& -384 p^{2}+3 p \cdot\left(p^{2}+8 p-4 d p+4 d^{2}-16 d+16\right) \cdot E(m, p) \\
= & (5 d-24) p^{4}-\left(5 d^{3}-48 d^{2}+180 d-320\right) p^{3} \\
& +\left(d^{5}-12 d^{4}+60 d^{3}-160 d^{2}+240 d-384\right) p^{2}+64 d p \\
& +3 p \cdot\left(p^{2}+8 p-4 d p+4 d^{2}-16 d+16\right) \cdot E(m, p) \\
= & U(p, d)+V(p, d) \cdot E(m, p) .
\end{aligned}
$$

This proves Lemma 3. 


\section{Proofs of the Theorems}

Now, we shall complete the proofs of our main results. Firstly, we prove Theorem 1 . Let $p$ be an odd prime with
$3 \mid p-1, \chi$ be any Dirichlet character modulo $p$, and $\lambda$ be a cubic character modulo $p$. Then, from the properties of the classical Gauss sums and (17), we have

$$
\begin{aligned}
& \sum_{\chi \bmod p}|G(m, 3, \chi ; p)|^{4}=\sum_{\chi \bmod p}\left|\sum_{a=1}^{p-1} \chi(a) e\left(\frac{m a^{3}}{p}\right)\right|^{4} \\
& =\sum_{\chi \bmod p} \sum_{a=1}^{p-1} \sum_{b=1}^{p-1} \sum_{c=1}^{p-1} \sum_{d=1}^{p-1} \chi(a b \bar{c} \bar{d}) e\left(\frac{m\left(a^{3}+b^{3}-c^{3}-d^{3}\right)}{p}\right) \\
& =(p-1) \sum_{\substack{a=1 \\
a b=c}}^{p-1} \sum_{\substack{b=1 \\
a b \bmod p)}}^{p-1} \sum_{\substack{d=1 \\
p-1}}^{p-1}\left(\frac{m\left(a^{3}+b^{3}-c^{3}-d^{3}\right)}{p}\right)
\end{aligned}
$$

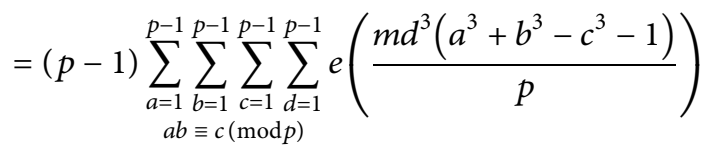

$$
\begin{aligned}
& =(p-1) \sum_{a=1}^{p-1} \sum_{b=1}^{p-1} \sum_{d=1}^{p-1} e\left(\frac{m d^{3}\left(a^{3}-1\right)\left(b^{3}-1\right)}{p}\right) \\
& =(p-1) \sum_{a=1}^{p-1} \sum_{b=1}^{p-1} \sum_{d=1}^{p-1}(1+\lambda(d)+\bar{\lambda}(d)) e\left(\frac{m d\left(a^{3}-1\right)\left(b^{3}-1\right)}{p}\right) \\
& =(p-1) \sum_{a=1}^{p-1} \sum_{b=1}^{p-1} \sum_{d=1}^{p-1} e\left(\frac{m d\left(a^{3}-1\right)\left(b^{3}-1\right)}{p}\right)+(p-1) \bar{\lambda}(m) \tau(\lambda) \sum_{a=1}^{p-1} \sum_{b=1}^{p-1} \bar{\lambda}\left(\left(a^{3}-1\right)\left(b^{3}-1\right)\right) \\
& +(p-1) \lambda(m) \tau(\bar{\lambda}) \sum_{a=1}^{p-1} \sum_{b=1}^{p-1} \lambda\left(\left(a^{3}-1\right)\left(b^{3}-1\right)\right) \\
& =(p-1) \sum_{a=1}^{p-1} \sum_{b=1}^{p-1} \sum_{d=1}^{p-1} e\left(\frac{m d\left(a^{3}-1\right)\left(b^{3}-1\right)}{p}\right)+(p-1) E(m, p),
\end{aligned}
$$

where $E(m, p)$ is the same as in Lemma 2 .

For any integer $n$, we have the trigonometric identity

$$
\sum_{a=1}^{p-1} e\left(\frac{n a}{p}\right)= \begin{cases}p-1, & \text { if }(n, p)=p, \\ -1, & \text { if }(n, p)=1 .\end{cases}
$$


From (30), we have the identity

$$
\begin{aligned}
& \sum_{a=1}^{p-1} \sum_{b=1}^{p-1} \sum_{d=1}^{p-1} e\left(\frac{m d\left(a^{3}-1\right)\left(b^{3}-1\right)}{p}\right) \\
& =2(p-1) \sum_{a=1}^{p-1} \sum_{\substack{b=1 \\
a^{3} \equiv 1(\bmod p)}}^{p-1} 1-(p-1) \sum_{\substack{a=1 \\
a^{3} \equiv 1(\bmod p)}}^{p-1} \sum_{b=1}^{p-1} 1-\sum_{\substack{a=1 \\
a^{3} \equiv 1(\bmod p)}}^{p-1} \sum_{b=1}^{p-1} 1 \\
& b^{3} \equiv 1(\bmod p) b^{3} \equiv 1(\bmod p) \\
& =6(p-1)^{2}-9(p-1)-(p-4)^{2}=5 p^{2}-13 p-1 .
\end{aligned}
$$

Combining (29)-(31), we have

$$
\begin{aligned}
\sum_{\chi \bmod p}|G(m, 3, \chi ; p)|^{4}= & \sum_{\chi \bmod p}\left|\sum_{a=1}^{p-1} \chi(a) e\left(\frac{m a^{3}}{p}\right)\right|^{4} \\
& =(p-1)\left(5 p^{2}-13 p-1\right)+(p-1) E(m, p) .
\end{aligned}
$$

For any positive integer $n$, from Lemma 3 , we have where

$E^{n}(m, p)=E^{3}(m, p) \cdot E^{n-3}(m, p)$

$$
\begin{aligned}
& =[U(p, d)+V(p, d) \cdot E(m, p)] \cdot E^{n-3}(m, p) \\
& =U(p, d) \cdot E^{n-3}(m, p)+V(p, d) \cdot E^{n-2}(m, p),
\end{aligned}
$$

$$
\begin{aligned}
U(p, d)= & 64 d p+\left(d^{5}-12 d^{4}+60 d^{3}-160 d^{2}+240 d-384\right) p^{2} \\
& -\left(5 d^{3}-48 d^{2}+180 d-320\right) p^{3}+(5 d-24) p^{4} \\
V(p, d)= & 3 p \cdot\left(p^{2}+8 p-4 d p+4 d^{2}-16 d+16\right) .
\end{aligned}
$$

This proves Theorem 1 .

Now, we prove Theorem 2. From Theorem 1, we have

$$
\sum_{m=1}^{p-1} E^{n}(m, p)=U(p, d) \sum_{m=1}^{p-1} E^{n-3}(m, p)+V(p, d) \sum_{m=1}^{p-1} E^{n-2}(m, p) .
$$


From Lemma 2, we have

$$
\begin{aligned}
\sum_{m=1}^{p-1} E(m, p)= & \sum_{m=1}^{p-1}\left(\bar{\lambda}(m) \tau(\lambda)\left(\frac{\tau^{3}(\bar{\lambda})}{p}-2\right)^{2}+\lambda(m) \tau(\bar{\lambda})\left(\frac{\tau^{3}(\lambda)}{p}-2\right)^{2}\right)=0 \\
\sum_{m=1}^{p-1} E^{2}(m, p)= & \sum_{m=1}^{p-1}\left(\bar{\lambda}(m) \tau(\lambda)\left(\frac{\tau^{3}(\bar{\lambda})}{p}-2\right)^{2}+\lambda(m) \tau(\bar{\lambda})\left(\frac{\tau^{3}(\lambda)}{p}-2\right)^{2}\right)^{2} \\
= & \sum_{m=1}^{p-1} \lambda(m) \tau^{2}(\lambda)\left(\frac{\tau^{3}(\bar{\lambda})}{p}-2\right)^{4}+\sum_{m=1}^{p-1} \bar{\lambda}(m) \tau^{2}(\bar{\lambda})\left(\frac{\tau^{3}(\lambda)}{p}-2\right)^{4} \\
& +\sum_{m=1}^{p-1} 2 p\left(\frac{\tau^{3}(\bar{\lambda})}{p}-2\right)^{2}\left(\frac{\tau^{3}(\lambda)}{p}-2\right)^{2} \\
= & 2 p(p-1)(p-2 d+4)^{2}
\end{aligned}
$$

From (36) and Lemma 3, we have

$$
\begin{aligned}
\sum_{m=1}^{p-1} E^{3}(m, p) & =\sum_{m=1}^{p-1}\left(\bar{\lambda}(m) \tau(\lambda)\left(\frac{\tau^{3}(\bar{\lambda})}{p}-2\right)^{2}+\lambda(m) \tau(\bar{\lambda})\left(\frac{\tau^{3}(\lambda)}{p}-2\right)^{2}\right)^{3} \\
& =\sum_{m=1}^{p-1}(U(p, d)+V(p, d) E(m, p))=(p-1) U(p, d) .
\end{aligned}
$$

Now, Theorem 2 follows from (35)-(38).

Finally, we prove Theorem 3 . For any integer $n \geq 1$, from Lemma 3, we have

$$
\begin{aligned}
\sum_{m=1}^{p-1} E^{-n}(m, p) & =\frac{1}{U(p, d)} \cdot \sum_{m=1}^{p-1} E^{-(n-3)}(m, p)-\frac{V(p, d)}{U(p, d)} \cdot \sum_{m=1}^{p-1} E^{-(n-1)}(m, p), \\
E^{2}(m, p) & =U(p, d) \cdot E^{-1}(m, p)+V(p, d), \\
E(m, p) & =U(p, d) \cdot E^{-2}(m, p)+V(p, d) \cdot E^{-1}(m, p), \\
E^{0}(m, p) & =U(p, d) \cdot E^{-3}(m, p)+V(p, d) \cdot E^{-2}(m, p), \\
E^{-1}(m, p) & =U(p, d) \cdot E^{-4}(m, p)+V(p, d) \cdot E^{-3}(m, p) .
\end{aligned}
$$


Therefore, we have

$$
\begin{aligned}
\sum_{m=1}^{p-1} E^{-1}(m, p) & =\sum_{m=1}^{p-1} \frac{E^{2}(m, p)-V(p, d)}{U(p, d)} \\
& =\frac{2 p(p-1)(p-2 d+4)^{2}-(p-1) V(p, d)}{U(p, d)}, \\
\sum_{m=1}^{p-1} E^{-2}(m, p) & =\sum_{m=1}^{p-1} \frac{E(m, p)-V(p, d) \cdot E^{-1}(m, p)}{U(p, d)} \\
& =\frac{(p-1) V^{2}(p, d)-2 p(p-1)(p-2 d+4)^{2} V(p, d)}{U^{2}(p, d)}, \\
\sum_{m=1}^{p-1} E^{-3}(m, p) & =\sum_{m=1}^{p-1} \frac{1-V(p, d) \cdot E^{-2}(m, p)}{U(p, d)} \\
& =\frac{p-1}{U(p, d)}+\frac{2 p(p-1)(p-2 d+4)^{2} V^{2}(p, d)-(p-1) V^{3}(p, d)}{U^{3}(p, d)}
\end{aligned}
$$

Combining (39)-(43), we may immediately complete the proof of Theorem 3.

\section{Conclusion}

The main results of this paper give two third-order linear recurrence formulae for the error term of the fourth power mean of the generalized cubic Gauss sums, and these results are the improvement and generalization of [4]. They are some new contributions in the relevant fields.

\section{Data Availability}

No data were used to support this study.

\section{Conflicts of Interest}

The authors declare that there are no conflicts of interest regarding the publication of this paper.

\section{Authors' Contributions}

All authors have equally contributed to this work. All authors read and approved the final manuscript.

\section{Acknowledgments}

This work was funded by the N. S. F. (11771351) of P. R. China and Xi'an Science and Technology Plan Innovation Fund "Special Project for Xi'an University" 2020KJWL08.

\section{References}

[1] T. Cochrane and Z. Zheng, "Pure and mixed exponential sums," Acta Arithmetica, vol. 91, no. 3, pp. 249-278, 1999.
[2] A. Weil, "On some exponential sums," Proceedings of the National Academy of Sciences of the United States of America, vol. 34, pp. 203-210, 1948.

[3] W. P. Zhang, "Moments of generalized quadratic Gauss sums weighted by L-functions," Journal of Number Theory, vol. 92, pp. 304-314, 2002.

[4] W. P. Zhang and H. N. Liu, "On the general Gauss sums and their fourth power mean," Osaka Journal of Mathematics, vol. 42, pp. 189-199, 2005.

[5] S. Chowla, J. Cowles, and M. Cowles, "On the number of zeros of diagonal cubic forms," Journal of Number Theory, vol. 9, no. 4 , pp. 502-506, 1977.

[6] T. M. Apostol, Introduction to Analytic Number Theory, Springer-Verlag, New York, NY, USA, 1976.

[7] K. Ireland and M. Rosen, A Classical Introduction to Modern Number Theory, Springer-Verlag, New York, NY, USA, 1982.

[8] C. D. Pan and C. B. Pan, Goldbach Conjecture, Science Press, Beijing, China, 1992.

[9] W. P. Zhang and J. Y. Hu, "The number of solutions of the diagonal cubic congruence equation mod $p$," Mathematical Reports, vol. 20, pp. 70-76, 2018.

[10] B. C. Berndt and R. J. Evans, "The determination of Gauss sums," Bulletin of the American Mathematical Society, vol. 5, no. 2, pp. 107-130, 1981. 